Am J Prev Med. 2018 February ; 54(2): 173-180. doi:10.1016/j.amepre.2017.11.008.

\title{
Screening for Alcohol Misuse: Practices Among U.S. Primary Care Providers, DocStyles 2016
}

\author{
Cheryl H. Tan, MPH, Daniel W. Hungerford, DrPH, Clark H. Denny, PhD, and Lela R. \\ McKnight-Eily, PhD \\ Centers for Disease Control and Prevention, Atlanta, Georgia
}

\begin{abstract}
Introduction-Excessive alcohol use is associated with car crashes, violence, and multiple disease conditions, including fetal alcohol spectrum disorders. The U.S. Preventive Services Task Force (USPSTF) recommends that primary care providers screen all adults and conduct brief counseling interventions with those who misuse alcohol. The USPSTF prefers use of three screening tools that measure alcohol consumption (Alcohol Use Disorders Identification Test, Alcohol Use Disorders Identification Test-Consumption, and National Institute on Alcohol Abuse and Alcoholism Single Question) because these tools detect the full spectrum of alcohol misuse in adults. This study estimated the prevalence of primary care provider screening practices for alcohol misuse and examined factors associated with using a USPSTF-preferred screening tool.
\end{abstract}

Methods-In 2016, a cross-sectional analysis was conducted on self-reported 2016 DocStyles data to estimate the prevalence of different screening tools used by 1,506 primary care providersfamily practitioners, internists, obstetrician/gynecologists, and nurse practitioners. Adjusted prevalence ratios were calculated using logistic regression to examine the association between provider attributes and use of USPSTF-preferred screening tools.

Results-In this study, $96 \%$ of providers reported screening patients for alcohol misuse. Among those that screened, 38\% used a USPSTF-preferred screening tool. Provider specialty, awareness of USPSTF guidelines, and mode of administering screening tool were associated with using a preferred screening tool.

Conclusions-Although most primary care providers reported screening for alcohol misuse, about two thirds did not use a tool capable of detecting the full spectrum of alcohol misuse. Using suitable screening tools will better identify patients who misuse alcohol and increase the opportunity for appropriate intervention, ultimately helping to reduce the burden from the many conditions associated with excessive alcohol consumption.

\section{INTRODUCTION}

Excessive alcohol consumption is associated with car crashes, violence, and more than 60 disease conditions, ${ }^{1}$ including hypertension, sleep disorders, various cancers, liver cirrhosis, and alcohol use disorders. ${ }^{2-4}$ In addition, maternal drinking during pregnancy can result in

Address correspondence to: Clark H. Denny, PhD, Centers for Disease Control and Prevention, 4770 Buford Highway NE, MS E86, Atlanta GA 30341. cdenny@cdc.gov. 
fetal alcohol spectrum disorders in the baby, ${ }^{5}$ and is associated with other poor birth outcomes, such as miscarriage, stillbirth, preterm birth, and sudden infant death syndrome. ${ }^{6}$ Reducing the burden from the health conditions associated with excessive drinking requires a focus on prevention - specifically, not only identifying patients with alcohol-related harm and alcohol use disorders, but also patients who are drinking in excess. ${ }^{7,8}$

For the purpose of this study, the term excessive alcohol use includes exceeding single-day limits (more than four drinks for men and more than three drinks for women in a single day), binge drinking (greater than single-day limits within a 2-hour period), exceeding weekly limits (more than 14 drinks for men and more than seven drinks for women per week), and any drinking by pregnant women or people under age 21 years. ${ }^{9,10}$ Estimates from 2009 to 2011 found that the prevalence of excessive drinking in the U.S. was $29.3 \%$ and the prevalence of alcohol dependence was $3.5 \%$; these estimates indicate that nondependent excessive drinkers outnumber dependent drinkers by more than eight to one. ${ }^{11}$

In 2004, the U.S. Preventive Services Task Force (USPSTF) found "good evidence that screening in primary care settings can accurately identify patients whose levels or patterns of alcohol consumption do not meet criteria for alcohol dependence, but place them at risk for increased morbidity and mortality" and that brief behavioral counseling interventions help reduce their alcohol consumption. ${ }^{12}$ This recommendation included a list of screening instruments for clinical use. Some of these screening tools, including the widely used CAGE (acronym for the four questions of the screening tool: Cut down, Annoyed, Guilty, Eye opener) questionnaire ${ }^{13-15}$ — which evaluates alcohol-related harm and alcohol dependence symptoms only, ${ }^{8}$ and not alcohol consumption-may be appropriate for detecting alcohol abuse and dependence, but are less capable of identifying non-dependent patients who drink too much. ${ }^{13,16}$ In 2013, the USPSTF updated their recommendation indicating a preference for three screening instruments that evaluated alcohol consumption and could, therefore, identify the full spectrum of alcohol misuse, defined as excessive alcohol use, alcohol abuse, and alcohol dependence. ${ }^{9}$ The tools had adequate sensitivity and specificity to identify which patients should receive a brief intervention. ${ }^{13}$ The three tools, which can be administered in 5 minutes or less, are the ten-question Alcohol Use Disorders Identification Test (AUDIT), ${ }^{17}$ the three-question AUDIT-Consumption (AUDIT-C), ${ }^{18}$ and single question screeners such as the National Institute on Alcohol Abuse and Alcoholism Single Question (NIAAA Single Question). ${ }^{19}$

Surveys among primary care providers (PCPs) and residents find a range of estimates for the prevalence of alcohol screening and use of different screening tools. Based on a 2010-2012 survey, $60 \%$ of medical residents from family and internal medicine programs in the Southeastern U.S. reported screening new patients for alcohol consumption, and $19 \%$ used tools that were capable of detecting excessive alcohol consumption. ${ }^{20}$ Compared with residents, providers reported a higher prevalence of screening in nationwide surveys, ranging from $85 \%$ to $88 \%,{ }^{14,21}$ with a majority not using a formal screening tool. ${ }^{15,21,22}$ When they used a validated instrument, they most commonly used the CAGE questionnaire. ${ }^{14,15}$ All of these studies were based, however, on data collected before the updated 2013 USPSTF recommendations. Moreover, because these studies do not estimate the prevalence of using specific screening tools, the percentage of providers using a tool capable of identifying the 
full spectrum of alcohol misuse behaviors cannot be determined. To fill these gaps, this study aims to describe current practices reported by PCPs when screening for alcohol misuse and to determine what demographics and practice characteristics are associated with using USPSTF-preferred screening tools.

\section{METHODS}

\section{Study Sample}

DocStyles is a survey developed by Porter Novelli with input from federal agencies and nonprofit and for-profit clients, which examines the knowledge, attitudes, and behaviors reported by healthcare providers in the U.S. Medical professionals registered with SERMO (an online global social network consisting of $>350,000$ medical professionals) were invited to be a part of the 2016 DocStyles survey. SERMO membership is free for medical professionals. Porter Novelli was responsible for setting sample size quotas in the following specialties: internists, family practitioners, obstetrician/gynecologists (OB/GYNs), and nurse practitioners. Healthcare professionals were eligible for the survey if they practiced in the U.S., actively saw patients, worked in individual/ group/hospital practices, and had been practicing for $\geq 3$ years. For the web-based survey, 2,384 respondents were invited to participate in June 2016; 1,506 respondents completed the survey (480 family practitioners, 523 internists, $250 \mathrm{OB} / \mathrm{GYNs}$, and 253 nurse practitioners). Respondents were paid an honorarium of $\$ 42-\$ 90$, depending on the number of questions they were asked. The overall response rate was $63.2 \%$ and differed by specialty (nurse practitioners $41.3 \%$, OB/GYNs $71.4 \%$, family practitioners and internists $70.5 \%$ ). Comparing internists, family practitioners, and OB/GYNs in this sample to the American Medical Association Physician Masterfile, this sample had a higher percentage of males (sample: 70\%, American Medical Association: 63\%) and had a lower mean for years in practice (sample: 17 years, American Medical Association: 22 years).

This research was exempt from IRB approval because the data file provided by Porter Novelli did not include personal identifiers.

\section{Measures}

DocStyles has a set of standard questions about provider demographics (age, gender, race/ ethnicity) and practice characteristics (specialty, inpatient/outpatient setting, years in practice, patients per week, practice size, region, financial situation of patients). These were included as potential covariates.

To ascertain screening practices for alcohol misuse, respondents were asked three questions. First, providers were asked whether alcohol screening and brief intervention (SBI) is a clinical preventive service recommended by the USPSTF. Second, they were asked to select all screening tools they used from the following list: AUDIT/AUDIT-C, CAGE, CRAFFT (acronym for the six questions of the screening tool: Car, Relax, Alone, Forget, Family or friends, Trouble), $T-A C E$ (acronym for the four questions of the screening tool: Tolerance, Annoyance, Cut down, Eye opener), TWEAK (acronym for the five questions of the screening tool: Tolerance, Worried, Eye opener, Amnesia, Cut down), NIAAA Single 
Question, ask number of drinks per occasion, ask frequency of drinking, don't use formal screening tool, or don't screen. The survey question included a definition of the NIAAA Single Question, specifically, How many times in the past year have you had 4 (women)/5 (men) or more drinks in a day? Third, respondents who reported screening using a formal or informal tool were asked whether screening questions were administered via in-person interview, form, electronically, or using other methods.

A binary dependent variable indicating whether the respondent used a preferred USPSTF screening tool was constructed from the question about screening tools. Respondents who selected the AUDIT/AUDIT-C or the NIAAA Single Question or both were classified as using a USPSTF-preferred screening tool.

\section{Statistical Analysis}

Analyses were conducted in 2016 using SAS-callable SUDAAN, version 11.0.1. Descriptive statistics of provider demographics and practice characteristics were presented for each specialty and for all four specialties combined. Responses to the three questions about screening for alcohol misuse were also presented for each specialty and for all four specialties combined. Bivariate analyses were conducted to estimate the prevalence of using a USPSTF-preferred screening tool by provider demographics, practice characteristics, and screening practices. Unadjusted $p$-values from chi-square tests were used to determine which variables had significant $(p \unlhd 0.05$ ) bivariate associations. Provider demographics commonly included in studies using DocStyles data, and covariates with significant associations in bivariate analyses, were included in a multivariate logistic regression to obtain adjusted prevalence ratios (APRs) and their corresponding 95\% CIs. The APRs and their CIs were similar for this parsimonious model and a model (not shown) that included all 11 potential covariates in Tables 1 and 2, providing evidence for robustness and lack of overfitting. The model was assessed for multicollinearity using condition indices and variance decomposition factors and no multicollinearity was found.

\section{RESULTS}

Within the sample, most PCPs were male (60\%), white non-Hispanic (66\%), worked in group outpatient practices (65\%), and saw $\geq 100$ patients per week (56\%), but differences existed by PCP specialty (Table 1). In particular, $88 \%$ of nurse practitioners were female, and only $26 \%$ saw $\geq 100$ patients per week. A majority of nurse practitioners and internists worked in practices with more than five providers, whereas a majority of OB/ GYNs and family practitioners worked in practices with five or fewer providers. Age differences also existed between specialties.

Most PCPs surveyed (81\%) were aware that the USPSTF recommends alcohol screening and brief counseling for all adults and most (96\%) reported screening adult patients for alcohol misuse (Table 2). However, most did not report using a USPSTF-preferred screening tool: $56 \%$ used the CAGE, $46 \%$ asked about drinking frequency, and $42 \%$ asked about number of drinks per occasion. Compared with other PCP specialties, OB/GYNs reported the highest prevalence of using other screening methods (20\%), T-ACE (10\%), and TWEAK $(4 \%)$. The most common mode of administering screening tools was via in-person interview, 
with $63 \%$ of providers reporting screening via in-person interview only, and another $18 \%$ reporting screening via in-person interview and form. By contrast, only $11 \%$ of providers screened via form only.

Among providers in this study who reported screening patients (96\%), the prevalence of using a USPSTF-preferred screening tool was 38\% (Table 3). Bivariate analyses showed that the following variables were significantly associated with using a USPSTF-preferred screening tool: provider specialty $(p<0.001)$, size of practice ( $p=0.041)$, awareness of the USPSTF recommendation on alcohol SBI ( $p=0.003)$, and mode through which screening questions were administered $(p<0.001)$. Controlling for these variables and provider demographics in a multivariate model showed that the prevalence of using a USPSTFpreferred screening tool was significantly lower among OB/GYNs than other specialties. In the same multivariate model, use of a USPSTF-preferred screening tool was $22 \%$ higher among providers aware of the USPSTF recommendation on alcohol SBI (APR=1.22, 95\% $\mathrm{CI}=1.02,1.47), 56 \%$ higher among providers who administered screening questions via inperson interview compared with form only ( $\mathrm{APR}=1.56,95 \% \mathrm{CI}=1.17,2.07$ ), and $152 \%$ higher among providers who administered screening questions via in-person interview and form compared with form only (APR=2.52, 95\% CI=1.88, 3.37).

\section{DISCUSSION}

This study describes current alcohol misuse screening practices reported by a sample of U.S. PCPs, and is the first study examining providers' reported use of USPSTF-preferred screening tools. Similar to the prevalence of provider-reported tobacco screening, ${ }^{23,24}$ almost all PCPs surveyed reported screening patients for alcohol misuse. However, only two in five providers (38\%) who screened used a USPSTF-preferred screening tool. This means that most PCPs surveyed were willing to ask patients about their alcohol use, but more than half may not be using screening tools capable of detecting the full spectrum of alcohol misuse, especially patients who drink excessively, but do not meet criteria for alcohol dependence. Predictors of using a preferred screening tool include physician specialty, awareness of the USPSTF recommendation on alcohol SBI, and mode of administering screening questions.

PCPs who do not screen for the full spectrum of alcohol misuse are likely to miss identifying many patients who could benefit from a brief intervention. A brief intervention is typically a 6-minute to 15 -minute interaction between the patient and provider about motivation to reduce or stop alcohol consumption and potential steps that would help. 9,25 Research shows that multiple-session brief interventions are effective at reducing weekly alcohol consumption, reducing heavy episodic drinking, and improving adherence to single day and weekly limits. ${ }^{26}$ Screening and brief intervention delivered electronically through computers, telephones, and mobile devices are also effective at reducing excessive alcohol consumption. ${ }^{27}$ The small percentage of patients who suffer from a severe alcohol use disorder can be referred for specialized treatment following brief intervention or treated in the primary care setting with medication-assisted treatment. ${ }^{19}$ 
The overall prevalence of screening in this study (96\%) was slightly higher than estimates from earlier studies based on provider surveys $(85 \%-88 \%) .{ }^{14,21}$ The higher prevalence in this study could be because DocStyles asked providers to select from a list of specific screening methods, which could have facilitated better recall, rather than asking generally about whether they screened patients. The differences could also be because of differences in sampling methodologies and populations. More research is needed to determine whether the differences reflect actual increases in the prevalence of screening for alcohol misuse in the past decade.

Studies based on patient surveys found that $25 \%-78 \%$ of patients received alcohol screening in person or by form. ${ }^{28,29} \mathrm{In}$ addition to differences in sampling methodologies, the lower estimates from patient surveys than provider surveys may be because of under-reporting among patients or over-reporting among providers. Specifically, patient surveys may be subject to greater recall bias because patients might only be screened at their first visit or during an annual checkup, whereas provider surveys may be subject to greater social desirability bias. It may also be a result of providers screening using a form and never communicating the results with their patients.

Although no study looked at predictors of using USPSTF-preferred screening tools, previous studies $^{21,22}$ found that knowledge of guidelines and provider specialty were predictors of using a formal screening tool in general. Specifically, knowledge about NIAAA drinking guidelines predicted using a formal screening tool among doctors and nurse practitioners in a managed care organization, ${ }^{22}$ and internists were more likely to use formal screening tools than family physicians or OB/GYNs. ${ }^{21}$ The lower use of formal or USPSTF-preferred tools among OB/GYNs might be because OB/GYNs are interested in establishing whether or not their pregnant patient drinks any alcohol. However, USPSTF-preferred tools assess quantity and frequency of alcohol consumption, making them suitable tools for OB/GYNs to screen both pregnant and non-pregnant patients. ${ }^{13}$ Further research is warranted to determine whether provider specialty and asking screening questions via in-person interview are consistently associated with using formal screening tools and, in particular, formal tools capable of detecting the full spectrum of alcohol misuse.

\section{Limitations}

The study is subject to at least six limitations. First, DocStyles respondents may not be representative of providers within their specialty. Moreover, data were not weighted so the percentages reported for the total sample may not be nationally representative. For these reasons, the results might not be generalizable to all U.S. PCPs. Second, the survey relied on providers' self-report, making it subject to social desirability and recall bias. Third, the question about choice of screening tools did not list all formal alcohol screening tools as options for the respondents. As such, the other screening method category could have included formal tools like the Michigan Alcohol Screening Test,${ }^{30}$ the Alcohol-Related Problems Survey, ${ }^{31}$ or even multi-drug screening tools like the Alcohol, Smoking and Substance Involvement Screening Test. ${ }^{32}$ Because these tools are not USPSTF-preferred screening tools, this limitation has little impact on this study's findings. Fourth, providers that reported using CAGE and asking patients about the number of drinks per occasion and 
frequency of drinking could potentially detect patients who misuse alcohol, even though they did not use a USPSTF-preferred screening tool. However, this combination of tools was not included because it does not have a formal threshold for identifying patients who misuse alcohol and because the provider could have used each tool on separate occasions rather than in combination. Fifth, the list of screening tools in the survey did not include the USAUDIT and USAUDIT1-3, which are updated versions of the AUDIT and AUDIT-C. ${ }^{33,34}$ These updated tools could reduce potential misclassification from using the AUDIT-C $\mathrm{C}^{35}$ by accounting for the larger standard drink size in the U.S. (14 $\mathrm{g}$ instead of $10 \mathrm{~g}$ of alcohol for international standard drinks), ${ }^{17}$ and lower NIAAA drinking limits among women and older men (www.niaaa.nih.gov/alcohol-health/overview-alcohol-consumption/moderate-bingedrinking). Lastly, the cross-sectional nature of the survey made it impossible to determine causal relationships, only predictors of using a USPSTF-preferred screening tool. Despite these limitations, this is the first study assessing the prevalence of using USPSTF-preferred screening tools among providers, based on the updated 2013 USPSTF recommendations.

\section{CONCLUSIONS}

Although almost all providers in the study reported screening patients for alcohol misuse, a majority of these providers who screen do not use a validated tool that measures alcohol consumption and, therefore, may not be able to identify the large number of patients who drink too much, but are not dependent on alcohol. Because nondependent excessive drinkers outnumber dependent drinkers by more than eight to one, ${ }^{11}$ this represents a significant missed opportunity for prevention. Using suitable screening tools will allow providers to better identify patients with alcohol misuse behaviors so that they can conduct appropriate clinical interventions. In addition to clinical strategies, community-level prevention strategies, such as increasing alcohol taxes, limiting alcohol outlet density, and limiting days or hours of alcohol sales, are effective at reducing excessive alcohol consumption. ${ }^{36}$ Applying both clinical and community-level strategies can reduce the burden from the many conditions associated with excessive alcohol consumption.

\section{Acknowledgments}

The findings and conclusions in this report are those of the authors and do not necessarily represent the official position of the Centers for Disease Control and Prevention.

Preliminary findings from this study were presented at the 7th International Conference on Fetal Alcohol Spectrum Disorders.

No financial disclosures were reported by the authors of this paper.

\section{References}

1. Rehm J, Room R, Graham K, Monteiro M, Gmel G, Sempos CT. The relationship of average volume of alcohol consumption and patterns of drinking to burden of disease: an overview. Addiction. 2003; 98(9):1209-1228. https://doi.org/10.1046/j.1360-0443.2003.00467.x. [PubMed: 12930209]

2. WHO. Global Status Report on Alcohol and Health 2014. Geneva, Switzerland: WHO; 2014.

3. Rehm J, Baliunas D, Borges GL, et al. The relation between different dimensions of alcohol consumption and burden of disease: an overview. Addiction. 2010; 105(5):817-843. https://doi.org/ 10.1111/j.1360-0443.2010.02899.x. [PubMed: 20331573] 
4. Ebrahim IO, Shapiro CM, Williams AJ, Fenwick PB. Alcohol and sleep I: effects on normal sleep. Alcohol Clin Exp Res. 2013; 37(4):539-549. https://doi.org/10.1111/acer.12006. [PubMed: 23347102]

5. Foltran F, Gregori D, Franchin L, Verduci E, Giovannini M. Effect of alcohol consumption in prenatal life, childhood, and adolescence on child development. Nutr Rev. 2011; 69(11):642-659. https://doi.org/10.1111/j.1753-4887.2011.00417.x. [PubMed: 22029831]

6. Bailey BA, Sokol RJ. Prenatal alcohol exposure and miscarriage, stillbirth, preterm delivery, and sudden infant death syndrome. Alcohol Res Health. 2011; 34(1):86-91. [PubMed: 23580045]

7. Institute of Medicine Committee on Treatment of Alcohol Problems. Broadening the Base of Treatment for Alcohol Problems. Washington, DC: National Academy Press; 1990.

8. Saunders JB, Aasland OG, Babor TF, de la Fuente JR, Grant M. Development of the Alcohol Use Disorders Identification Test (AUDIT): WHO Collaborative Project on Early Detection of Persons with Harmful Alcohol Consumption-II. Addiction. 1993; 88(6):791-804. https://doi.org/10.1111/j. 1360-0443.1993.tb02093.x. [PubMed: 8329970]

9. Moyer VA. U S. Preventive Services Task Force. Screening and behavioral counseling interventions in primary care to reduce alcohol misuse: U.S. Preventive Services Task Force recommendation statement. Ann Intern Med. 2013; 159(3):210-218. https://doi.org/ 10.7326/0003-4819-159-3-201308060-00652. [PubMed: 23698791]

10. U.S. DHHS, U.S. Department of Agriculture. [Accessed November 18, 2017] 2015-2020 Dietary Guidelines for Americans. 8https://health.gov/dietaryguidelines/2015/guidelines/. Published December 2015

11. Esser MB, Hedden SL, Kanny D, Brewer RD, Gfroerer JC, Naimi TS. Prevalence of alcohol dependence among U.S. adult drinkers, 2009-2011. Prev Chronic Dis. 2014; 11:E206. https:// doi.org/10.5888/pcd11.140329. [PubMed: 25412029]

12. U S. Preventive Services Task Force. Screening and behavioral counseling interventions in primary care to reduce alcohol misuse: recommendation statement. Ann Intern Med. 2004; 140(7):554 556. https://doi.org/10.7326/0003-4819-140-7-200404060-00016. [PubMed: 15068984]

13. Jonas, DE., Garbutt, JC., Brown, JM., et al. Screening, Behavioral Counseling, and Referral in Primary Care to Reduce Alcohol Misuse. Rockville, MD: Agency for Healthcare Research and Quality; 2012.

14. Barry KL, Blow FC, Willenbring ML, McCormick R, Brockmann LM, Visnic S. Use of alcohol screening and brief interventions in primary care settings: implementation and barriers. Subst Abus. 2004; 25(1):27-36. https://doi.org/10.1300/J465v25n01_05.

15. Anderson BL, Dang EP, Floyd RL, Sokol R, Mahoney J, Schulkin J. Knowledge, opinions, and practice patterns of obstetrician-gynecologists regarding their patients' use of alcohol. J Addict Med. 2010; 4(2):114-121. https://doi.org/10.1097/ADM.0b013e3181b95015. [PubMed: 21769028]

16. Whitlock EP, Polen MR, Green CA, Orleans T, Klein J. U S. Preventive Services Task Force. Behavioral counseling interventions in primary care to reduce risky/harmful alcohol use by adults: a summary of the evidence for the U.S. Preventive Services Task Force. Ann Intern Med. 2004; 140(7):557-568. https://doi.org/10.7326/0003-4819-140-7-200-404060-00017. [PubMed: 15068985]

17. Babor, TF., Higgins-Biddle, JC., Saunders, JB., Monteiro, MG. AUDIT: The Alcohol Use Disorders Identification Test: Guidelines for Use in Primary Care. 2. Geneva, Switzerland: WHO; 2001.

18. Bush K, Kivlahan DR, McDonell MB, Fihn SD, Bradley KA. The AUDIT alcohol consumption questions (AUDIT-C): an effective brief screening test for problem drinking. Ambulatory Care Quality Improvement Project (ACQUIP). Alcohol Use Disorders Identification Test. Arch Intern Med. 1998; 158(16):1789-1795. https://doi.org/10.1001/archinte.158.16.1789. [PubMed: 9738608]

19. U.S. DHHS, National Institutes of Health, National Institute on Alcohol Abuse and Alcoholism. Helping Patients Who Drink Too Much: A Clinician's Guide. Rockville, MD: U.S. DHHS; 2005. NIH Publication No. 07-3769 
20. Le KB, Johnson JA, Seale JP, et al. Primary care residents lack comfort and experience with alcohol screening and brief intervention: a multi-site survey. J Gen Intern Med. 2015; 30(6):790796. https://doi.org/10.1007/s11606-015-3184-y. [PubMed: 25666210]

21. Friedmann PD, McCullough D, Chin MH, Saitz R. Screening and intervention for alcohol problems. A national survey of primary care physicians and psychiatrists. J Gen Intern Med. 2000; 15(2):84-91. https://doi.org/10.1046/j.1525-1497.2000.03379.x. [PubMed: 10672110]

22. Gassman RA. Practitioner-level predictors of alcohol problems detection and management activities. J Subst Use. 2007; 12(3):191-202. https://doi.org/10.1080/14659890701237215.

23. Tong EK, Strouse R, Hall J, Kovac M, Schroeder SA. National survey of U.S. health professionals' smoking prevalence, cessation practices, and beliefs. Nicotine Tob Res. 2010; 12(7):724-733. https://doi.org/10.1093/ntr/ntq071. [PubMed: 20507899]

24. Kruger J, O'Halloran A, Rosenthal A. Assessment of compliance with U.S. Public Health Service clinical practice guideline for tobacco by primary care physicians. Harm Reduct J. 2015; 12:7. https://doi.org/10.1186/s12954-015-0044-3. [PubMed: 25889679]

25. McKnight-Eily LR, Liu Y, Brewer RD, et al. Vital signs: communication between health professionals and their patients about alcohol use-44 states and the District of Columbia, 2011. MMWR Morb Mortal Wkly Rep. 2014; 63:16-22. [PubMed: 24402468]

26. Jonas DE, Garbutt JC, Amick HR, et al. Behavioral counseling after screening for alcohol misuse in primary care: a systematic review and meta-analysis for the U.S. Preventive Services Task Force. Ann Intern Med. 2012; 157(9):645-654. https://doi.org/ 10.7326/0003-4819-157-9-201211060-00544. [PubMed: 23007881]

27. Community Preventive Services Task Force. Alcohol electronic screening and brief intervention: recommendation of the Community Preventive Services Task Force. Am J Prev Med. 2016; 51(5): 812-813. https://doi.org/10.1016/j.amepre.2016.04.012. [PubMed: 27745679]

28. Denny CH, Hungerford DW, McKnight-Eily LR, et al. Self-reported prevalence of alcohol screening among U.S. adults. Am J Prev Med. 2016; 50(3):380-383. https://doi.org/10.1016/ j.amepre.2015.09.016. [PubMed: 26520573]

29. McKnight-Eily LR, Okoro CA, Mejia R, et al. Screening for excessive alcohol use and brief counseling of adults-17 states and the District of Columbia, 2014. MMWR Morb Mortal Wkly Rep. 2017; 66(12):313-319. https://doi.org/10.15585/mmwr.mm6612a1. [PubMed: 28358798]

30. Selzer ML. The Michigan alcoholism screening test: the quest for a new diagnostic instrument. Am J Psychiatry. 1971; 127(12):1653-1658. https://doi.org/10.1176/ajp.127.12.1653. [PubMed: 5565851]

31. Fink A, Morton SC, Beck JC, et al. The alcohol-related problems survey: identifying hazardous and harmful drinking in older primary care patients. J Am Geriatr Soc. 2002; 50(10):1717-1722. https://doi.org/10.1046/j.1532-5415.2002.50467.x. [PubMed: 12366628]

32. WHO Assist Working Group. The Alcohol, Smoking and Substance Involvement Screening Test (ASSIST): development, reliability and feasibility. Addiction. 2002; 97(9):1183-1194. https:// doi.org/10.1046/j.1360-0443.2002.00185.x. [PubMed: 12199834]

33. CDC. Planning and Implementing Screening and Brief Interventions for Risky Alcohol Use: A Step-by-Step Guide for Primary Care Practices. Atlanta, GA: CDC, National Center on Birth Defects and Developmental Disabilities; 2014.

34. Babor, TF., Higgins-Biddle, JC., Robaina, K. The Alcohol Use Disorders Identification Test, Adapted for Use in the United States: A Guide for Primary Care Practitioners. Rockville, MD: Substance Abuse and Mental Health Services Administration; 2016.

35. Delaney KE, Lee AK, Lapham GT, Rubinsky AD, Chavez LJ, Bradley KA. Inconsistencies between alcohol screening results based on AUDIT-C scores and reported drinking on the AUDITC questions: prevalence in two U.S. national samples. Addict Sci Clin Pract. 2014; 9:2. https:// doi.org/10.1186/1940-0640-9-2. [PubMed: 24468406]

36. Task Force on Community Prevention Services. [Accessed August 13, 2017] Evidence-based strategies to prevent excessive alcohol consumption and related harm. www.thecommunityguide.org/sites/default/files/assets/ Summary_Alcohol_Interventions082413.pdf. Published 2013 


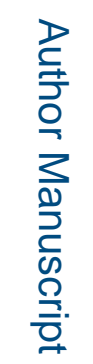

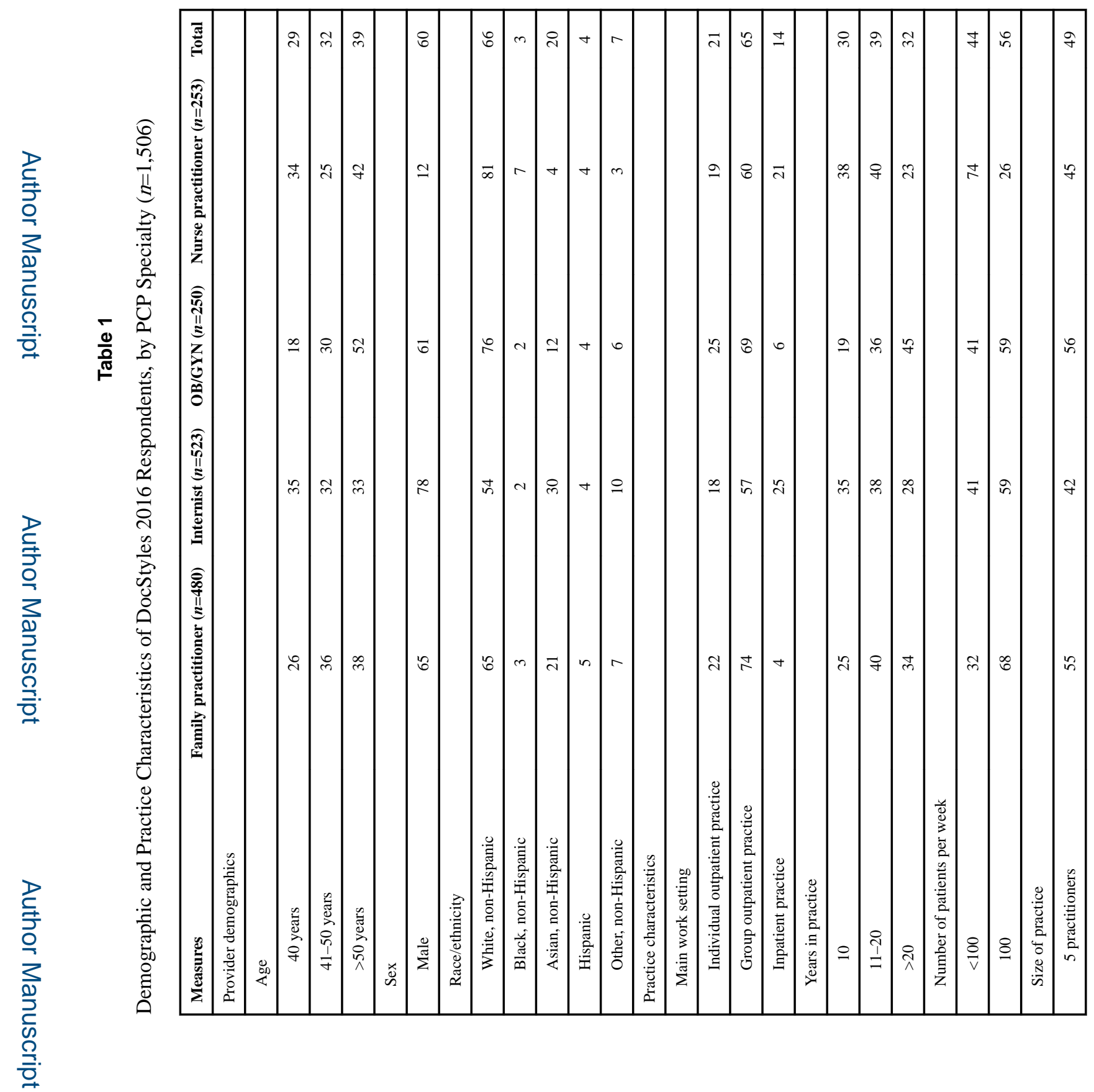

Am J Prev Med. Author manuscript; available in PMC 2019 February 01. 


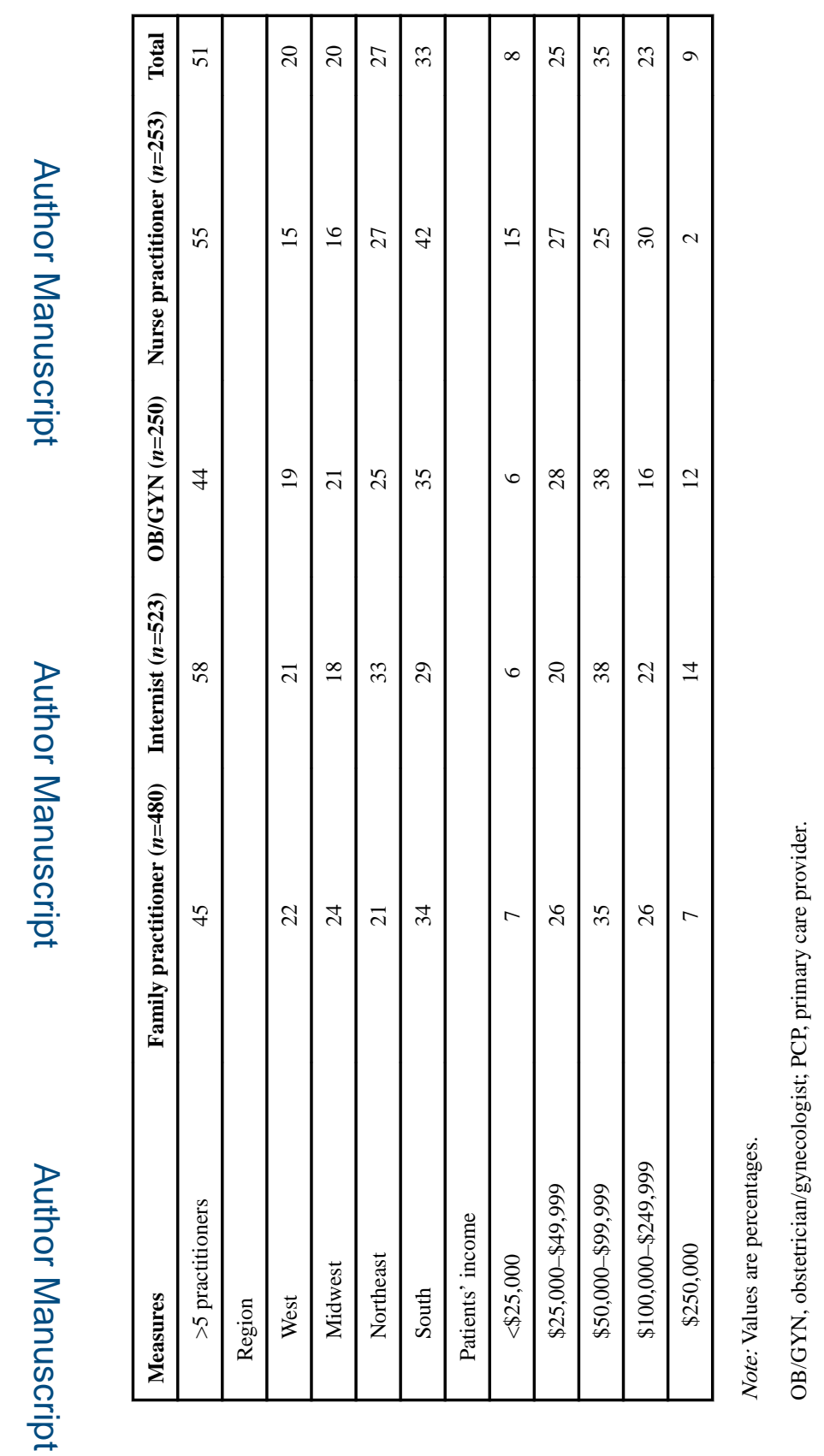

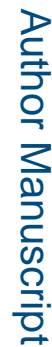




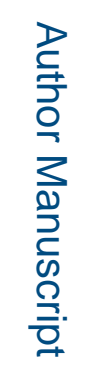

棓

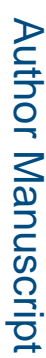
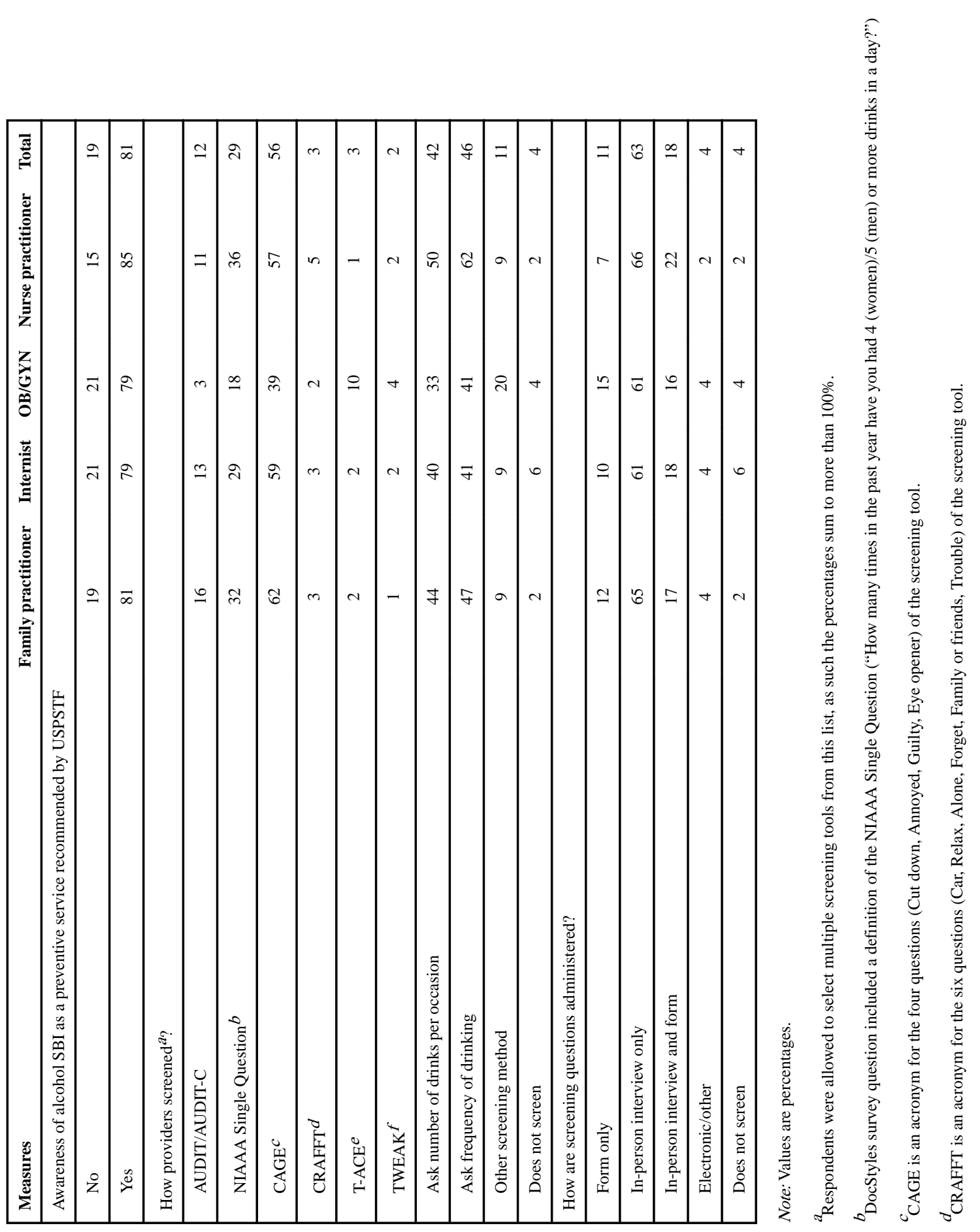

Am J Prev Med. Author manuscript; available in PMC 2019 February 01. 


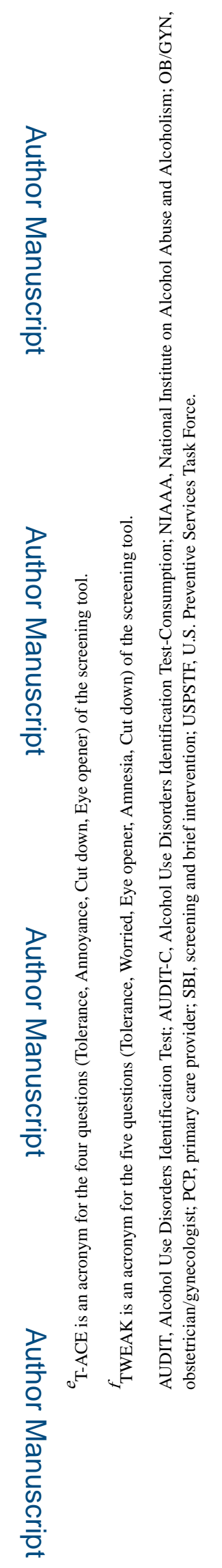

Am J Prev Med. Author manuscript; available in PMC 2019 February 01. 
Table 3

Selected Characteristics Associated With Using USPSTF-Preferred Screening Tools Among PCPs Who Screen, DocStyles $2016(n=1,434)$

\begin{tabular}{|c|c|c|c|c|}
\hline Measures & Prevalence (\%) & Unadjusted $\chi^{2} p$-value & $\mathbf{A P R}^{a}$ & $95 \% \mathrm{CI}$ \\
\hline Overall & 38 & & & \\
\hline \multicolumn{5}{|l|}{ Provider demographics } \\
\hline Age & & 0.681 & & \\
\hline$\leq 40$ years & 39 & & ref & \\
\hline $41-50$ years & 39 & & 1.09 & $0.93,1.28$ \\
\hline$>50$ years & 37 & & 1.07 & $0.90,1.26$ \\
\hline Gender & & 0.273 & & \\
\hline Male & 39 & & 1.11 & $0.96,1.29$ \\
\hline Female & 37 & & ref & \\
\hline Race/ethnicity & & 0.227 & & \\
\hline White, non-Hispanic & 38 & & 1.34 & $0.86,2.08$ \\
\hline Black, non-Hispanic & 30 & & ref & \\
\hline Asian, non-Hispanic & 42 & & 1.40 & $0.88,2.22$ \\
\hline Hispanic & 28 & & 1.06 & $0.60,1.87$ \\
\hline Other, non-Hispanic & 40 & & 1.43 & $0.87,2.34$ \\
\hline \multicolumn{5}{|l|}{ Practice characteristics $b$} \\
\hline Specialty & & $<0.001$ & & \\
\hline Family practitioner & 45 & & 2.16 & $1.66,2.81$ \\
\hline Internist & 40 & & 1.83 & $1.40,2.39$ \\
\hline OB/GYN & 20 & & ref & \\
\hline Nurse practitioner & 41 & & 1.98 & $1.48,2.66$ \\
\hline Size of practice & & 0.041 & & \\
\hline$\$ 5$ practitioners & 36 & & ref & \\
\hline$>5$ practitioners & 41 & & 1.13 & $0.99,1.29$ \\
\hline $\begin{array}{l}\text { Awareness of alcohol SBI as a preventive service recommended by the } \\
\text { USPSTF }\end{array}$ & & 0.003 & & \\
\hline Yes & 40 & & 1.22 & $1.02,1.47$ \\
\hline No & 30 & & ref & \\
\hline How are screening questions administered? & & $<0.001$ & & \\
\hline Form only & 22 & & ref & \\
\hline In-person interview only & 36 & & 1.56 & $1.17,2.07$ \\
\hline Form and interview & 59 & & 2.52 & $1.88,3.37$ \\
\hline Electronic/other & 21 & & 0.91 & $0.52,1.60$ \\
\hline
\end{tabular}

Note: Boldface indicates statistical significance $(p<0.05)$.

${ }^{a}$ Adjusted for all other covariates in the model. 


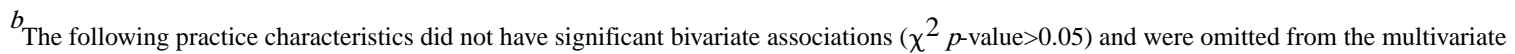
model: main work setting, years in practice, number of patients per week, region, and financial situation of patients.

APR, adjusted prevalence ratio; PCP, primary care provider; ref, reference; SBI, screening and brief intervention; USPSTF, U.S. Preventive Services Task Force. 